\title{
PROPOSTA DE UM SISTEMA DE MEDIÇÃO DE DESEMPENHO APLICADO À MECANIZAÇÃ O AGRÍCOLA
}

\section{PAULO R. PELOIA ${ }^{1}$, MARCOS MILAN ${ }^{2}$}

RESUMO: As organizações líderes dos mais diferentes setores têm como característica medir o próprio desempenho de modo sistêmico, porém nas empresas agrícolas ainda não é comum o emprego desse conceito, incluindo o setor de mecanização. A mecanização tem um forte impacto na composição dos custos de produção, e conhecer o seu desempenho é primordial para o sucesso de empresa agrícola. A importância que as medidas de desempenho têm no auxílio à gestão e o que a mecanização representa para os custos de produção justificaram o desenvolvimento deste trabalho, que tem como objetivo propor um sistema de medição de desempenho integrado para dar suporte à gestão da mecanização agrícola. A metodologia foi dividida em duas etapas: adaptação de um modelo conceitual sistêmico, com base no Balanced Scorecard - BSC; aplicação do modelo a um estudo de caso na agroindústria canavieira. A adaptação e a aplicação do modelo conceitual permitiu obter indicadores de desempenho de modo sistêmico e associados a: custo e prazo (tradicionalmente utilizados); controle e melhoria na qualidade de operações e processos de apoio; preservação ambiental; segurança, saúde, satisfação, motivação e capacitação de colaboradores; desenvolvimento de sistemas de informação. Conclui-se que o modelo auxiliou na elaboração do sistema de medição de desempenho para a gestão de sistemas mecanizados e que os indicadores permitem uma visão integrada da empresa e associada aos objetivos estratégicos.

PALAVRAS-CHAVE: máquinas agrícolas, mecanização agrícola, sistemas de informação, BSC.

\section{PROPOSAL OF A PERFORMANCE MEASURING SYSTEM APPLIED TO AGRICULTURAL MECHANIZATION}

\begin{abstract}
The leaders' organizations of several different sectors have as characteristic to measure their own performance in a systematic way. However, this concept is still unusual in agricultural enterprises, including the mechanization sector. Mechanization has an important role on the production costs and to know its performance is a key factor for the agricultural enterprise success. This work was generated by the importance that measurement of performance has for the management and the mechanization impact on the production costs. Its aim is to propose an integrated performance measurement system to give support to agricultural management. The methodology was divided in two steps: adjustment of a conceptual model based on Balanced Score Card - BSC; application of the model in a study case at sugar cane mill. The adjustment and the application of the conceptual model allowed to obtain the performance index in a systematic way, that are associated to: costs and deadline (traditionally used); control and improvement on the quality of operations and support process; environmental preservation; safety; health; employees satisfaction; development of information systems. The adjusted model helped the development of the performance measurement system for the mechanized management systems and the index allows an integrated view of the enterprise, related to its strategic objectives.
\end{abstract}

KEYWORDS: agricultural machinery, agricultural mechanization, information systems, BSC.

\footnotetext{
${ }^{1}$ Eng $^{\text {}}$ Agronômo, Mestre em Máquinas Agrícolas pela ESALQ/USP, prpeloia@uol.com.br.

${ }^{2}$ Professor do Departamento de Engenharia de Biossistemas da ESALQ/USP, macmilan@esalq.usp.br. 


\section{INTRODUÇÃO}

Quando se estudam as organizações que se mantêm líderes por longos períodos e nos mais diferentes setores, uma das características sempre presentes é a habilidade delas em medir o seu próprio desempenho e de usar a medição para buscar patamares superiores. As organizações que não dispõem de um sistema de medição de desempenho estruturado são mais vulneráveis às mudanças no ambiente externo. Normalmente, essas organizações têm o seu sistema de medição mais voltado para indicadores financeiros que não refletem a verdadeira realidade nem garantem a sustentabilidade (FPNQ, 2002). Um sistema de medição de desempenho é um conjunto de indicadores inter-relacionados entre si, que têm como objetivo principal controlar e auxiliar na tomada de decisões, do nível estratégico ao operacional. Nesse sentido, o controle é uma das forças fundamentais que mantêm a organização eficiente (SALAZAR, 2001).

KAPLAN \& NORTON (1997) desenvolveram um sistema de medição de desempenho denominado de Balanced Scorecard (BSC). De acordo com os autores, o método pode ser aplicado para grandes corporações, joint-ventures, departamentos de apoio, unidades de negócio, empresas públicas e filantrópicas. As principais características do BSC são: uso de poucos indicadores; equilíbrio entre indicadores financeiros e não financeiros; relação causa e efeito. $\mathrm{O}$ uso de poucos indicadores relacionados diretamente à estratégia empresarial mantém o foco e não permite que a empresa evolua mais em determinado ponto em detrimento de outro, de forma não previsível. $\mathrm{O}$ equilíbrio dos indicadores financeiros e não financeiros (clientes, processos internos e inovação e crescimento), faz com que seja possível a gestão de ativos intangíveis relacionados a tendências futuras, pois os indicadores financeiros relatam, basicamente, o que já aconteceu. Relacionar os indicadores na forma de causa e efeito permite que seja possível entender a influência esperada que um ativo intangível possa ter sobre os tangíveis. Os autores descrevem que o sistema permite respostas a quatro questões básicas para a organização: "como o cliente nos vê - perspectiva do cliente; em que devemos atingir a excelência- perspectiva interna da empresa; como acentuar e gerar valor continuamente- perspectiva da inovação e aprendizado; que imagem os acionistas fazem da empresa- perspectiva financeira".

Segundo FPNQ (2002), um modelo de medição de desempenho de uma organização deve ter como base os seguintes conceitos e critérios: permitir a visão sistêmica e integrada; refletir a lógica da estratégia; usar de forma pró-ativa o sistema de indicadores; incorporar todos os níveis hierárquicos; apresentar consequências claras do desempenho associado com resultados.

$\mathrm{Na}$ agricultura brasileira, a mecanização agrícola representa um fator de grande importância para a competitividade em termos de custo, chegando a ser o segundo fator de produção mais importante, sendo inferior apenas à posse da terra. Em termos de potencial para redução dos custos de produção, a mecanização pode ser considerada como o fator principal. Para se reduzirem os custos são necessários a ampliação e a modernização da gestão dos sistemas mecanizados. A adoção de técnicas administrativas clássicas não atende mais às condições de sustentabilidade impostas pelo mercado. Essas técnicas têm como fundamento o dimensionamento do sistema mecanizado, os estudos dos tempos e movimentos e o planejamento e controle de custos e produtividade. Elas são eficazes em momentos de menor concorrência, mas desprovidas de visão sistêmica. A adequação do gerenciamento de sistemas mecanizados deve buscar, além de produtividade e custos, qualidade de operações agrícolas, motivação, segurança e saúde dos funcionários, preservação do ambiente e alinhamento estratégico (MILAN, 2004). Como suporte a essa adequação rumo à sustentabilidade, é fundamental o desenvolvimento de medidas do desempenho referentes aos sistemas mecanizados que permitam a visão sistêmica e integrada.

$\mathrm{Na}$ área agrícola, os principais estudos abordam a medição de desempenho por meio da análise individual de indicadores. A maior parte destes trabalhos visa a aplicar na agricultura metodologias inicialmente utilizadas nas indústrias, resultando em indicadores operacionais para o controle do trabalho do dia a dia. Como trabalhos relacionados a esse tipo de indicadores na área agrícola podem ser citados: LOPES et al. (1995), FERNANDES et al. (2000), MILAN \& 
FERNANDES (2002), PECHE FILHO et al. (2002), PINTO (2002), MILAN et al. (2003), BONATO (2004), NAGUMO \& MILAN (2006), CAMPOS (2007), NUINTIN (2007), PELOIA \& MILAN (2007), SALVI et al. (2007) e SUGUISAWA et al. (2007).

Considerando-se que a eficácia da gestão dos sistemas mecanizados é um fator importante para o sucesso das empresas agrícolas e que um sistema de medição de desempenho, de caráter sistêmico, pode contribuir para esse sucesso, o objetivo deste trabalho é propor um sistema de medição de desempenho integrado para dar suporte à gestão da mecanização agrícola

\section{MATERIAL E MÉTODOS}

A metodologia adotada, referente à proposta de um sistema de medição de desempenho para a mecanização agrícola, foi dividida em duas etapas: elaboração de um modelo conceitual e aplicação como estudo de caso. O desenvolvimento foi realizado com base na adaptação do modelo conceitual do BSC- Balanced Scorecard, proposto por KAPLAN \& NORTON (1997) e nos critérios e conceitos de FPNQ (2002). O estudo de caso foi realizado junto a uma empresa, agroindústria canavieira produtora de bioenergia.

A empresa (usina) está localizada na região de Pereira Barreto - SP, região noroeste paulista, é produtora de álcool, açúcar e energia elétrica. Para atender a essa atividade de produção, a empresa conta com um sistema mecanizado agrícola com aproximadamente 350 equipamentos entre máquinas agrícolas, caminhões de transporte, colhedoras e implementos. Esses equipamentos são empregados na produção, colheita e transporte da matéria-prima, cana-de-açúcar, para a usina.

As fases propostas pelo BSC seguidas até a proposição das medidas de desempenho são apresentadas na Figura 1. Elas correspondem a: Fase 1 - política básica e objetivos estratégicos; Fase 2 - objetivos da área funcional; Fase 3 - fatores críticos de sucesso; Fase 4 - mapa estratégico (nível gerencial); Fase 5 - indicadores gerenciais.

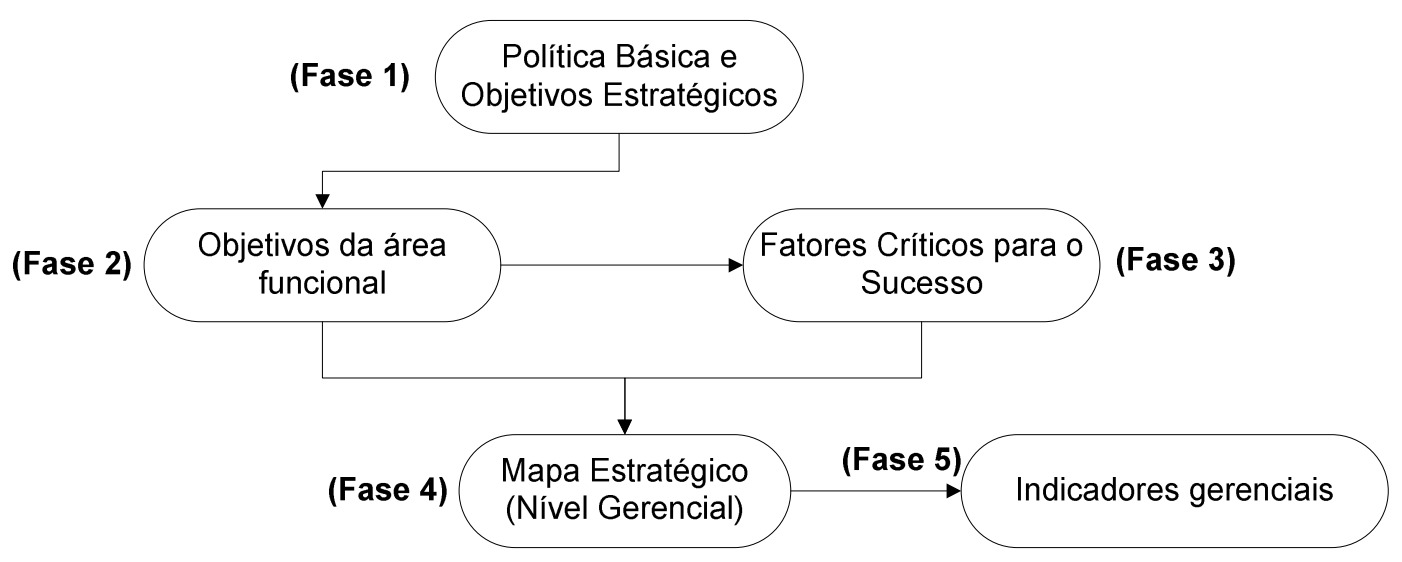

FIGURA 1. Fases do trabalho. Research stages.

A Fase 1 (Figura 1) foi realizada junto à direção e à gerência agrícola e tem como início o levantamento da política básica (missão, visão e valores) e dos objetivos estratégicos da empresa. A política e os objetivos devem nortear os objetivos da área funcional. No caso deste trabalho, o levantamento das informações versou sobre o que é esperado da gerência de mecanização agrícola, para que esta gerência contribua com os objetivos estratégicos da empresa. Essas informações constituem os objetivos da área funcional, no caso o sistema mecanizado agrícola, Fase 2. Os fatores críticos de sucesso FCS, pontos vitais de uma organização para seu sucesso futuro, foram desenvolvidos para cada objetivo da área funcional, Fase 3. Esse desenvolvimento foi realizado por meio de entrevistas não estruturadas, de acordo com a proposta de ROCHA (1991), com os diretores (proprietários), gerentes, coordenadores e corpo técnico da organização estudada, corpo técnico da empresa, análise do banco de dados e da documentação e observação direta. Com base 
nos fatores, desenvolveu-se o mapa estratégico do nível gerencial (Fase 4), como forma de representar graficamente a estratégia e facilitar sua visualização e compreensão. A partir do mapa estratégico, são propostos os indicadores gerenciais de resultados associados aos objetivos (Fase 5) e tendência, associados aos FCS. Os indicadores foram validados junto ao corpo técnico da empresa.

Em função da proposta deste trabalho, propor uma metodologia, os indicadores não serão apresentados em sua totalidade, mas apenas aqueles considerados como de maior relevância para este propósito no caso, indicadores pertencentes à perspectiva de processos internos. A escolha dos processos internos segue a recomendação de KAPLAN \& NORTON (1997) para organizações cuja estratégia global segue a linha de liderança do custo. A empresa em questão busca a competitividade pela redução no custo de produção do açúcar, álcool e energia. Como a empresa não detém o controle do preço de venda, os indicadores de maior importância estão na perspectiva de processos internos. Embora com foco no processo interno, o mesmo raciocínio pode ser aplicado no desenvolvimento dos indicadores para as demais perspectivas: financeira, clientes e inovação e conhecimento.

Durante o desenvolvimento do trabalho, utilizou-se como base para o desenvolvimento alguns indicadores já existentes. Destacam-se aqui os trabalho de GONÇALVES et al. (1993), que propõem uma metodologia básica para medir a eficiência de uso de máquinas, o de SOFFNER \& BALASTREIRE (1994), que descrevem os indicadores da qualidade de manutenção de uma frota, o de BRITO \& BRITO (2000) que descrevem o índice de Qualidade de Vida (QVT), índice que pondera fatores que afetam o bem-estar biológico, psicológico e social dos colaboradores, e o de RAMOS (2000) com relação à qualidade de processos, medida por índices de capacidade do processo.

\section{RESULTADOS E DISCUSSÃO}

Os resultados do desenvolvimento do modelo referentes aos objetivos estratégicos (Fase 1), objetivos da área funcional (Fase 2) e fatores críticos de sucesso (Fase 3) são representados no mapa estratégico (Fase 4), Figura 2.

O mapa é dividido em duas partes principais. Os objetivos relacionados com a redução de custos são apresentados no lado esquerdo, estratégia de produtividade, e no lado direito, os objetivos relacionados com a sustentabilidade ambiental e social, estratégia de sustentabilidade. A estratégia de produtividade envolve basicamente ações que visem à otimização da frota e à realização das operações de produção e apoio com qualidade e dentro do prazo, estando relacionada à melhoria da rotina do dia a dia. Por sua vez, a estratégia de sustentabilidade envolve ações relacionadas com a expansão dos negócios e oportunidades envolvendo as mudanças no sistema de produção, avaliação na seleção de insumos e ferramentas (máquinas) utilizadas.

Os objetivos estratégicos do sistema mecanizado agrícola da perspectiva financeira visam a aumentar a produtividade dos recursos utilizados, redução de entradas e incremento de saídas, com resultados visíveis no curto prazo, e a expansão das oportunidades de negócios alcançando clientes mais exigentes, por meio da adoção de técnicas sustentáveis, com resultados de médio e longo prazos.

A perspectiva de cliente tem como objetivos o atendimento das exigências de qualidade e prazo dos clientes internos, representados pelo departamento industrial e agrícola, e construir uma boa imagem da empresa para a sociedade, empresa responsável, com relação a cuidados com a preservação do ambiente e condições de segurança e saúde dos colaboradores. A perspectiva de processos internos busca a excelência operacional em processos-chave de produção e apoio, contribuindo tanto para a perspectiva financeira (custos) e de clientes (prazo, qualidade, preservação do ambiente, segurança e saúde dos colaboradores). A perspectiva de inovação e conhecimento tem como objetivos estratégicos o desenvolvimento de: sistemas de informação; cultura organizacional (melhoria contínua); desenvolvimento de colaboradores (gestão da qualidade, melhoria de 
processos e crescimento do ser humano); desenvolvimento de tecnologias de produção com práticas voltadas para a produção ambientalmente sustentável (limpas).

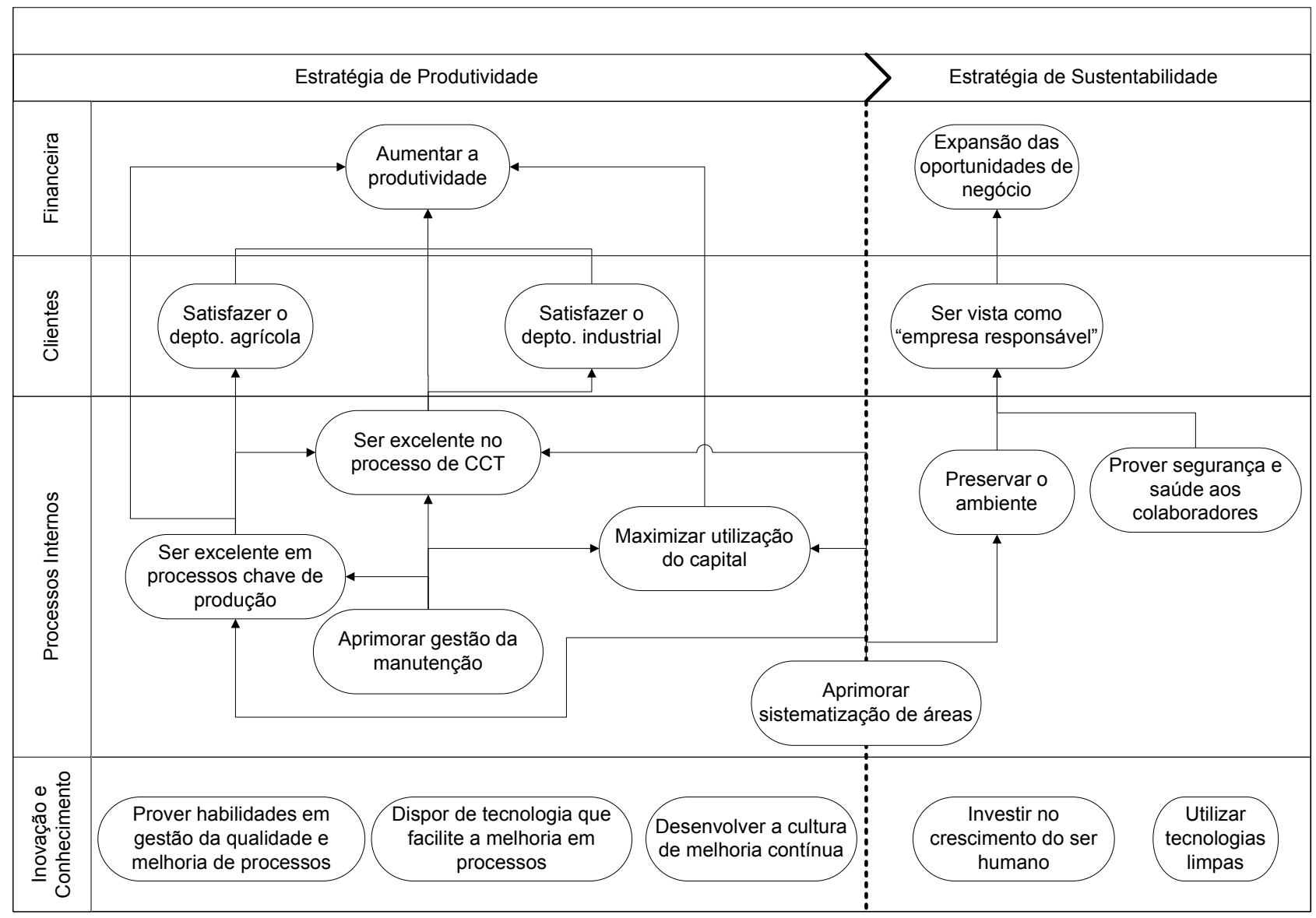

FIGURA 2. Mapa estratégico da gestão de sistemas mecanizados agrícolas da organização estudada. Strategic chart of an agricultural mechanized system management.

\section{Perspectiva de processos internos da estratégia de produtividade}

Para que a satisfação dos clientes e acionistas possa ser atingida, é necessário que os processos internos críticos da empresa estejam direcionadas para este fim. Nos processos de produção de cana, apenas a produção de mudas não é realizada pelo sistema mecanizado. $\mathrm{O}$ departamento agrícola tem sua satisfação associada diretamente ao sucesso das ações realizadas pelos processos-chave de produção de cana e o departamento industrial ao processo-chave de corte, carregamento e transporte (CCT).

Os processos-chave de produção e CCT têm como objetivos a excelência operacional, que consiste em realizar suas atividades da melhor maneira possível, equilibrando custo, qualidade e prazo (Figura 3), seus fatores críticos de sucesso.

Para o fator crítico relacionado com o custo, o objetivo dos processos é evitar o desperdício de insumos, principalmente produtos fitossanitários e combustíveis. Seus indicadores referem-se ao custo com insumos por área e de combustível por tonelada de cana.

O fator crítico qualidade no processo tem relação com a produtividade da cana e na qualidade da matéria-prima fornecida à usina pelo CCT. Os indicadores de qualidade buscam medir se os processos estão gerando produtos (ou serviços) que atendem às especificações feitas pelo cliente, especificações estas dadas por índices de capacidade do processo.

O fator crítico prazo, associado ao CCT, irá influenciar no abastecimento contínuo e na qualidade da cana fornecida para a indústria (deterioração por microrganismos da matéria-prima cana). Na produção, é fundamental que as operações agrícolas sejam cumpridas em um determinado 
prazo, sendo este um FCS para a produtividade e qualidade, para que o potencial produtivo da cultura possa ser expresso. Com relação ao indicador de eficiência para o processo de produção, o seu objetivo é medir o aproveitamento do tempo disponível para trabalho, que assim contribui para a execução das tarefas dentro do tempo previsto. Para o CCT, a eficiência de entrega de cana está associada à relação quantidade de cana entregue e a quantidade planejada- meta.

\begin{tabular}{|c|c|}
\hline Objetivo & Fator crítico de sucesso \\
\hline & $\begin{array}{l}\text { DEFINIÇÃO:Redução no uso de insumos } \\
\text { INDICADOR:Custo com insumos por área }\end{array}$ \\
\hline \multirow[t]{3}{*}{$\begin{array}{l}\text { DEFINIÇÃO:Ser excelente em processos-chave de } \\
\text { produção } \\
\text { INDICADOR:Indicador ponderado de excelência }\end{array}$} & $\begin{array}{l}\text { DEFINIÇÃO:Atendimento à qualidade } \\
\text { INDICADOR:\% da população estimada dentro da } \\
\text { tolerância }\end{array}$ \\
\hline & $\begin{array}{l}\text { DEFINIÇÃO:Cumprimento de prazos } \\
\text { INDICADOR:Eficiência global }\end{array}$ \\
\hline & $\begin{array}{l}\text { DEFINIÇÃO:Redução no uso de combustível } \\
\text { INDICADOR:Custo com combustível por tonelada de } \\
\text { cana }\end{array}$ \\
\hline \multirow[t]{2}{*}{$\begin{array}{l}\text { DEFINIÇÃO:Ser excelente em processos de CCT } \\
\text { INDICADOR:Indicador ponderado de excelência }\end{array}$} & $\begin{array}{l}\text { DEFINIÇÃO:Atendimento à qualidade } \\
\text { INDICADOR:\% da população estimada dentro da } \\
\text { tolerância }\end{array}$ \\
\hline & $\begin{array}{l}\text { DEFINIÇÃO:Cumprimento de prazos } \\
\text { INDICADOR:Eficiência de entrega de cana }\end{array}$ \\
\hline
\end{tabular}

FIGURA 3. Indicadores gerenciais da perspectiva interna para os processos-chave de produção e CCT. Management indexes from internal perspective for the production keyprocess and CCT.

Uma importante constatação realizada durante a realização do trabalho foi a influência da qualidade dos processos de produção de cana no processo de CCT. A qualidade dos processos de produção, além de estar diretamente ligada à satisfação do departamento agrícola, determinará a qualidade do processo de CCT e, consequentemente, a satisfação do cliente departamento industrial.

O objetivo do processo interno relacionado com a maximização do capital investido (Figura 4) não contribui para a satisfação dos clientes, porém está relacionado diretamente com a satisfação dos acionistas. Os seus FCSs estão relacionados com o dimensionamento correto da frota, de forma que as máquinas fiquem o menor tempo possível ociosas. Porém, o dimensionamento não deve reduzir a frota própria, na busca de reduzir o tempo ocioso, e compensar isso com o uso de serviços de terceiros, pois para a empresa em questão, os serviços realizados por terceiros apresentam valores mais elevados quando comparados ao realizado pela própria usina. Além disso, existe uma dificuldade maior para controlar a qualidade das operações. 


\begin{tabular}{|c|c|}
\hline Objetivo & Fator crítico de sucesso \\
\hline & DEFINIÇÃO: Maximização da utilização das máquinas \\
\hline \multirow{2}{*}{$\begin{array}{l}\text { DEFINIÇÃO: Maximizar utilização do capital } \\
\text { INDICADOR: Custo fixo horário de máquinas e } \\
\text { equipamentos }\end{array}$} & INDICADOR: Uso anual das máquinas \\
\hline & DEFINICÃO: Reducão da terceirizacão \\
\hline & INDICADOR: \% de horas-máquina terceirizadas \\
\hline
\end{tabular}

FIGURA 4. Indicadores gerenciais da perspectiva interna para a maximização da utilização do capital. Management indexes from internal perspective for capital profit maximization.

O custo fixo anual das máquinas e equipamentos, de acordo com MIALHE (1974), é composto pela depreciação, juros sobre o capital investido e alojamento, seguros e taxas. A razão entre o custo fixo anual e o número de horas trabalhadas resulta no custo fixo horário. Portanto, o custo fixo horário está associado diretamente com a utilização das máquinas. Os indicadores de tendência são o número de horas trabalhadas, como forma de medida do uso das máquinas, e o percentual de participação nas horas das máquinas terceirizadas, uma forma de medir a dependência de terceiros.

A manutenção da frota é outro fator de grande importância. Ela se relaciona com o custo da manutenção em si e da pontualidade, com a realização de atividades no período determinado e a qualidade (SOFFNER \& MIALHE, 1994). O gerenciamento da manutenção deve contribuir para o gerenciamento da frota e tem como objetivo garantir a disponibilidade dos equipamentos, de modo a atender ao processo de produção com confiabilidade, segurança, preservação do ambiente e custo adequado (PINTO \& XAVIER, 2001).

Para aprimorar a gestão da manutenção, um índice ponderado de excelência deve ser estabelecido com base no custo de manutenção e confiabilidade da frota e prazo de duração das quebras (Figura 5). Indicadores para a qualidade da manutenção são disponibilidade e confiabilidade. Além dos indicadores de qualidade, o custo de manutenção, que completa a composição dos custos variáveis proposta por MIALHE (1974), dado pelo custo por hora de operação, também possui grande importância.

\begin{tabular}{|l|l|}
\hline Objetivo & Fator crítico de sucesso \\
\hline
\end{tabular}

\begin{tabular}{|c|c|}
\hline & $\begin{array}{l}\text { DEFINIÇÃO: Redução de custos } \\
\text { INDICADOR: Custo horário com manutenção }\end{array}$ \\
\hline $\begin{array}{l}\text { DEFINIÇÃO: Aprimorar gestão da manutenção } \\
\text { INDICADOR: Indicador ponderado de excelência }\end{array}$ & $\begin{array}{l}\text { DEFINIÇÃO: Redução do número de quebras } \\
\text { INDICADOR: Confiabilidade }\end{array}$ \\
\hline & $\begin{array}{l}\text { DEFINIÇÃO: Redução no tempo de paradas por quebra } \\
\text { INDICADOR: Duração das quebras }\end{array}$ \\
\hline
\end{tabular}

FIGURA 5. Indicadores gerenciais da perspectiva interna para a gestão da manutenção. Management indexes from internal perspective indexes for the maintenance management.

O objetivo de melhorar a sistematização de áreas está relacionado com a melhor eficiência de uso dos tempos por parte dos processos de produção, por meio da redução do número de manobras 
(Figura 6). LOPES \& MILAN (1998) confirmam esta relação quando apontam para o processo de CCT a eficiência operacional como um fator importante na redução dos custos.

Outro ponto importante da sistematização de áreas é a redução do revolvimento do solo por meio da redução de operações de preparo, fazendo com que o solo fique o menor tempo possível sem cobertura vegetal, ficando assim menos suscetível a erosões.

\begin{tabular}{|l|l|}
\hline Objetivo & Fator crítico de sucesso \\
\hline
\end{tabular}

\begin{tabular}{|c|c|}
\hline & $\begin{array}{l}\text { DEFINIÇÃO: Redução da movimentação do solo } \\
\text { INDICADOR: Número médio de operações de preparo de } \\
\text { solo }\end{array}$ \\
\hline \multirow{2}{*}{$\begin{array}{l}\text { DEFINIÇÃO: Aprimorar sistematização de áreas } \\
\text { INDICADOR: \% de áreas que passaram no processo de } \\
\text { sistematização }\end{array}$} & DEFINIÇÃO: Redução do número de manobras \\
\hline & $\begin{array}{l}\text { INDICADOR: Eficiência de campo } \\
\text { INDICADOR: Distância vertical entre terraços }\end{array}$ \\
\hline
\end{tabular}

FIGURA 6. Indicadores gerenciais da perspectiva interna para sistematização de áreas. Management indexes from internal perspective for areas systematization.

$\mathrm{O}$ indicador de resultado tem o objetivo de medir o percentual das áreas que já sofreram a sistematização. O indicador de tendência para a movimentação do solo tem o objetivo de quantificar o revolvimento do solo, sendo feito por meio do número médio de operações realizadas até que o solo esteja preparado. Os indicadores de tendência, selecionados pela empresa, para quantificar o número de manobras foram dois: eficiência de campo e distância vertical entre terraços.

\section{Perspectiva de processos internos da estratégia de sustentabilidade}

Os indicadores atrelados à preservação do ambiente, saúde e segurança dos colaboradores são apresentados na Figura 7.

\begin{tabular}{|l|l|}
\hline Objetivo & Fator crítico de sucesso \\
\hline
\end{tabular}

\begin{tabular}{|c|c|}
\hline & DEFINIÇÃO:Redução de área de colheita de cana \\
\hline \multirow{4}{*}{$\begin{array}{l}\text { DEFINIÇÃO:Preservar o ambiente } \\
\text { INDICADOR:Indicador ponderado de preservação do } \\
\text { ambiente }\end{array}$} & INDICADOR:\% de área de cana colhida queimada \\
\hline & \\
\hline & INDICADOR: \% de óleo usado descartado corretamente \\
\hline & DEFINIÇÃO:Redução de exposição ao risco \\
\hline DEFINIÇÃO:Prover saúde e segurança aos & INDICADOR:Frequência e gravidade de acidentes \\
\hline INDICADOR:Indice de qualidade de vida no trabalhol & DEFINIÇÃO:Melhoria na ergonomia \\
\hline & INDICADOR: \% de pessoas com doença ocupacional \\
\hline
\end{tabular}

FIGURA 7. Indicadores gerenciais da perspectiva interna para preservação do ambiente. Management indexes from internal perspective for environment preservation. 
Para a preservação do ambiente, o indicador ponderado de tendência leva em conta o percentual da área de colheita de cana queimada e resíduos destinados incorretamente. Assim, esses indicadores têm o objetivo de medir o percentual da área colhida com queima prévia e o percentual de óleo lubrificante consumido que não é descartado corretamente. A principal preocupação com resíduos é a correta utilização do óleo das colhedoras mecanizadas. Verificou-se, por meio de um levantamento das ordens de serviço de manutenção realizadas nos equipamentos, uma constante recorrência de rompimentos de mangueiras do sistema hidráulico das colhedoras durante o trabalho e, consequentemente, perda de óleo no ambiente. A redução dos rompimentos das mangueiras permitirá destinar o óleo para a recuperação ao invés de liberá-lo no ambiente. A correta destinação de outros resíduos, como pneus, outros tipos de óleos lubrificantes e embalagens de agroquímicos, são considerados importantes pela empresa, porém já têm um processo de coleta satisfatório, não necessitando assim de um indicador específico.

O objetivo de prover segurança e saúde aos colaboradores está relacionado com a manutenção da integridade física, por meio da redução de exposição ao risco e a melhoria da ergonomia. O indicador selecionado foi o Índice de Qualidade de Vida (QVT). Os indicadores de tendência são a gravidade e a frequência de acidentes dado pelo número de acidentes e dias de afastamento por mil horas trabalhadas, sendo uma medida de exposição ao risco. O percentual de pessoas com doença ocupacional é uma medida da qualidade da ergonomia e condições do ambiente nas atividades.

\section{CONCLUSÕES}

O modelo do BSC mostrou-se capaz de auxiliar no desenvolvimento de um sistema de medição de desempenho para a mecanização agrícola.

Além de indicadores de custo e prazo, tradicionalmente utilizados e estudados, o sistema de medição de desempenho apontou indicadores para: controle e melhoria na qualidade de operações e processos de apoio, preservação ambiental, segurança, saúde, satisfação, motivação e capacitação de colaboradores; desenvolvimento de sistemas de coleta e difusão de dados.

Com base no sistema de medição, observa-se a necessidade da visão integrada de indicadores que são atrelados aos objetivos estratégicos da empresa, e as suas relações de causa e efeito. Desta forma, poucos indicadores essenciais são necessários para a gestão da mecanização agrícola.

\section{AGRADECIMENTOS}

A Pioneiros Bioenergia S.A., pelo suporte técnico e financeiro à pesquisa.

\section{REFERÊNCIAS}

BONATO, R.G. Qualidade operacional da fenação: análise do processo de produção. 2004. 100 f. Dissertação (Mestrado em Máquinas Agrícolas) - Escola Superior de Agricultura "Luiz de Queiroz", Universidade de São Paulo, Piracicaba, 2004.

BRITO; M. J. de; BRITO, V. da G.P. Gestão estratégica de recursos humanos. Lavras: UFLA/FAEPE, 2000. 47 p.

CAMPOS, C.M. de. Identificação de variáveis críticas no processo de produção de cana-de-açúcar. 2007. 88 f. Dissertação (Mestrado em Máquinas Agrícolas) - Escola Superior de Agricultura "Luiz de Queiroz", Universidade de São Paulo, Piracicaba, 2007.

FERNANDES, R.A.T.; MILAN, M.; PECHE FILHO, A. Gerenciamento da qualidade em operações mecanizadas em um sistema de produção de cana-de-açúcar. Engenharia Agrícola, Jaboticabal, v.20, n.3, p.215-220, 2000.

FUNDAÇÃO PARA O PRÊMIO NACIONAL DA QUALIDADE - FPNQ. Planejamento do Sistema de medição de desempenho: relatório do comitê temático. 2.ed. São Paulo, 2002. 96 p. 
GONÇALVES, N.G.; LIMA, E.B.; BANCHI, A. D.; PINTO, R.S.A.; LOPES, J.R. Eficiências de uso de máquina agrícola. In: CONGRESSO NACIONAL DA SOCIEDADE DE TÉCNICOS AÇUCAREIROS E ALCOOLEIROS DO BRASIL, 5., 1993, Águas de São Pedro. Anais... Piracicaba: STAB, 1993. p.165-168.

KAPLAN, R.S.; NORTON, D.P. A estratégia em ação: Balanced Scorecard. 21.ed. Tradução: FRAZÃO FILHO, L.E.T. Rio de Janeiro: Elsevier, 1997. 344 p.

LOPES, M.B.; MILAN, M.; COELHO, J.L.D. Qualidade em operações agrícolas mecanizadas na cultura da cana-de-açúcar. Stab Açúcar, Álcool e Subprodutos, Piracicaba, v.13, n.3, p.26-30, 1995.

LOPES, M.B.; MILAN, M. Simulation of a sugar cane loading and transport system. In: INTERNATIONAL CONFERENCE ON COMPUTERS IN AGRICULTURE, 7., 1998, Orlando. Proceedings... Orlando: ASAE, 1998. p.124-130.

MIALHE, L.G. Manual de mecanização agrícola. São Paulo: Agronômica Ceres, 1974. 301 p.

MILAN, M. Gestão sistêmica e planejamento de máquinas agrícolas. 2004. 100 f. Tese (Livre-Docência em Mecânica e Máquinas Agrícolas) - Escola Superior de Agricultura "Luiz de Queiroz", Universidade de São Paulo, Piracicaba, 2004.

MILAN, M.; BARROS, J.W.D.; GAVA, J.L. Planning soil tillage using quality function deployment (QFD). Scientia Agricola, Piracicaba, v.60, n.2, p.217-221, 2003.

MILAN, M.; FERNANDES, R.A.T. Qualidade das operações de preparo de solo por controle estatístico do processo. Scientia Agrícola, Piracicaba, v.59, n.2, p.261-266, 2002.

NAGUMO, G.K.; MILAN, M. Desdobramento da função qualidade (QFD) aplicado à produção de mudas de café (Coffea arabica L.). Engenharia Rural, Piracicaba, v.17, n.2, p.45-51, 2006.

NUINTIN, A.A. O desenvolvimento de indicadores do desempenho e da qualidade para o processo de produção: estudo de casos do processo de produção do café. 2007. 145 f. Dissertação (Mestrado em Controladoria e Contabilidade) - Faculdade de Economia, Administração e Contabilidade de Ribeirão Preto, Universidade de São Paulo, Ribeirão Preto, 2007.

PECHE FILHO, A.; SILVA, L.C.; BÊN, J.C.; NAGAOKA, A.K. Avaliação de qualidade de subsolagem com base em medidas de profundidade na reformulação de lavoura de cana-de-açúcar. In: CONGRESSO BRASILEIRO DE ENGENHARIA AGRÍCOLA, 31., 2002, Salvador. Anais... Salvador: Associação Brasileira de Engenharia Agrícola, 2002. 1 CD-ROM.

PELOIA, P.R.; MILAN, M. Indicadores de desempenho operacionais de uma frente de colheita mecanizada de cana-de-açúcar. In: CONGRESSO BRASILEIRO DE ENGENHARIA AGRÍCOLA, 36., 2007, Bonito. Anais... Bonito: Associação Brasileira de Engenharia Agrícola, 2007. 1 CD-ROM.

PINTO, R.S. de A. Indicadores de desempenho de frota de empresas agroindustriais canavieiras brasileiras. 2002. 110 f. Dissertação (Mestrado em Máquinas Agrícolas) - Escola Superior de Agricultura "Luiz de Queiroz”, Universidade de São Paulo, Piracicaba, 2002.

PINTO, A.K.; XAVIER, J.A.N. Manutenção: função estratégica. 2.ed. Rio de Janeiro: Qualimark, 2001. $341 \mathrm{p}$.

RAMOS, A.W. CEP para processos contínuos e em bateladas. São Paulo: Edgard Blucher/ Fundação Vanzolini, 2000. 130 p.

ROCHA, L.O.L. da. Organização e métodos: uma abordagem prática. São Paulo: Atlas, 1991. $286 \mathrm{p}$.

SALAZAR, G.T. Administração geral: teoria e gerência das organizações. Lavras: UFLA/FAEPE, 2001. 153 p. 
SALVI, J.V.; MATOS, M.A.; MILAN, M. Avaliação do desempenho de dispositivo de corte de base de colhedora de cana-de-açúcar. Engenharia Agrícola, Jaboticabal, v.27, n.1, p.201-209, 2007.

SOFFNER, R.K.; BALASTREIRE, L.A. Manutenção preditiva em sistemas mecanizados agrícolas. Engenharia Rural, Piracicaba, v.5, n.1, p.66-72, 1994.

SOFFNER, R.K.; MIALHE, L.G. Avaliação da confiabilidade de tratores agrícolas. Engenharia Rural, Piracicaba, v.5, n.1, p.54-65, 1994.

SUGUISAWA, J.M.; FRANCO, F.N.; SILVA, S.S.S.; PECHE FILHO, A. Qualidade de aplicação de herbicida em lavoura de trigo. Engenharia Agrícola, Jaboticabal, v.27, n.1, p.41-47, 2007. 